\title{
Periodic Solution for Stochastic Predator-Prey Systems with Nonlinear Harvesting and Impulses
}

\author{
Yafei Yang, Yuanfu Shao, Mengwei Li \\ College of Physics, Guilin University of Technology, Guilin, China \\ Email: 1115917086@qq.com, shaoyuanfu@163.com,419763620@qq.com
}

How to cite this paper: Yang, Y.F., Shao, Y.F. and Li, M.W. (2019) Periodic Solution for Stochastic Predator-Prey Systems with Nonlinear Harvesting and Impulses. $A d$ vances in Linear Algebra \& Matrix Theory, 9, 89-103.

https://doi.org/10.4236/alamt.2019.94007

Received: September 9, 2019

Accepted: November 12, 2019

Published: November 15, 2019

Copyright $\odot 2019$ by author(s) and Scientific Research Publishing Inc. This work is licensed under the Creative Commons Attribution International License (CC BY 4.0).

http://creativecommons.org/licenses/by/4.0/

\begin{abstract}
In this paper, astochastic predator-prey systems with nonlinear harvesting and impulsive effect are investigated. Firstly, we show the existence and uniqueness of the global positive solution of the system. Secondly, by constructing appropriate Lyapunov function and using comparison theorem with an impulsive differential equation, we study that a positive periodic solution exists. Thirdly, we prove that system is globally attractive. Finally, numerical simulations are presented to show the feasibility of the obtained results.
\end{abstract}

\section{Keywords}

Impulses Perturbations, Periodic Solution, Non-Linear Harvesting, Stochastic Predator-Prey Systems, Globally Attractive

\section{Introduction}

It is well known that the dynamic relationship between predator and prey has always been one of the main topics in ecology and mathematical ecology. In the past decades, many predator-prey models have been proposed and widely used to describe the food supply relationship between two species [1] [2]. At the same time, it has attracted great attention in many different fields, such as bio-economics. Recently, the interaction of predator-prey with harvesting has been studied. The effect of harvest on population is beneficial to sustainable development and renewable resource management, so many scholars take harvest into account in their models. The capture intensity depends largely on the capture strategy being implemented. Common harvest functions are: constant harvest, proportional harvest and nonlinear harvest. Gupta et al. proposed a predator-prey model with nonlinear predator in harvest [3] and discussed the 
dynamical properties of the following system:

$$
\left\{\begin{array}{l}
\mathrm{d} x=x(t)\left(r_{1}-b_{1} x(t)\right)-a x(t) y(t) \mathrm{d} t, \\
\mathrm{~d} y=y(t)\left(-r_{2}+\operatorname{\eta ax}(t) y(t)-\frac{h y(t)}{1+b y(t)}\right) \mathrm{d} t,
\end{array}\right.
$$

On the other hand, the growth of species in nature is often limited by environmental factors. Generally speaking, there are two main types of environmental noise: white noise and colored noise. Wenjie Zuo et al. [4] considered the white noise and studied the stationary distribution and periodic solution. However, reading the literature found that studies on the non-linear harvesting of predators and prey are very few literatures [5] [6] [7]. Therefore, the following model is proposed.

$$
\left\{\begin{aligned}
\mathrm{d} x(t)= & x(t)\left[r_{1}-a_{11}(t) x(t)-a_{12}(t) y(t)-\frac{H(t)}{1+b(t) x(t)}\right] \mathrm{d} t \\
& +\sigma_{1}(t) x(t) \mathrm{d} B_{1}(t), \\
\mathrm{d} y(t)= & y(t)\left[-r_{2}+a_{21}(t) x(t)-a_{22}(t) y(t)-\frac{h(t)}{1+b(t) y(t)}\right] \mathrm{d} t \\
& -\sigma_{2}(t) y(t) \mathrm{d} B_{2}(t)-\frac{\sigma_{3}(t) h(t) y(t)}{1+b(t) y(t)} \mathrm{d} B_{3}(t),
\end{aligned}\right.
$$

In real life, however, ecosystems are often disturbed by human development or by activities related to natural factors such as drought, floods, earthquakes, and planting. In order to describe this phenomenon more accurately, impulses perturbation is added into the model. To sum up, this paper mainly studies the effects of impulse effect and nonlinear harvesting on predator and prey populations, and proposes the following interesting stochastic system.

$$
\left\{\begin{aligned}
& \mathrm{d} x(t)= x(t)\left[r_{1}(t)-a_{11}(t) x(t)-a_{12}(t) y(t)-\frac{H(t)}{1+b(t) x(t)}\right] \mathrm{d} t \\
&+\sigma_{1}(t) x(t) \mathrm{d} B_{1}(t), \\
& \mathrm{d} y(t)= y(t)\left[-r_{2}(t)+a_{21}(t) x(t)-a_{22}(t) y(t)-\frac{h(t)}{1+b(t) y(t)}\right] \mathrm{d} t \\
&-\sigma_{2}(t) y(t) \mathrm{d} B_{2}(t)-\frac{\sigma_{3}(t) h(t) y(t)}{1+b(t) y(t)} \mathrm{d} B_{3}(t), \\
& x\left(t_{k}^{+}\right)-x\left(t_{k}\right)=\alpha_{k} x\left(t_{k}\right), y\left(t_{k}^{+}\right)-y\left(t_{k}\right)=\beta_{k} y\left(t_{k}\right), t=t_{k}, k=1,2,3, \cdots
\end{aligned}\right.
$$

where $x(t)$ and $y(t)$ represent the density of prey and predator populations respectively. The parameters $r_{i}(t), a_{i j}(t),(i, j=1,2)$ are positive, and $r_{1}$ is the internal growth rate of prey, and $r_{2}$ is the mortality rate of predator. $a_{11}(t)$ and $a_{22}(t)$ represent the intra-specific competition coefficients of prey and predator populations, respectively. The coefficient $a_{12}(t)$ is the predator's capture rate and $a_{21}(t)$ stands for the rate at which nutrients are converted to predators. In addition, $\frac{H(t)}{1+b(t) x(t)}, \frac{h(t)}{1+b(t) y(t)}$ are the nonlinear harvesting. 
Throughout this paper, unless otherwise specified, we suppose $\left(\Omega, \mathcal{F},\left\{\mathcal{F}_{t}\right\}_{t \geq 0}, \mathbb{P}\right)$ be a complete probability space with a filtration $\left\{\mathcal{F}_{t}\right\}_{t \geq 0}$ satisfying the usual conditions and it is right continuous and increasing, while $\mathcal{F}_{0}$ contains all $\mathbb{P}$ -null set. All the coefficients are assumed to be $T$-periodic continuous functions.

The remainder of this paper is organized as follows. In Section 2, we show that the model (1.3) existence of the global positive solution. In Section 3, sufficient conditions are achieved to guarantee the existence of a positive periodic solution of the stochastic system (1.3) by using Itô's formula. In Section 4, we discuss the globally attractive of stochastic model (1.3). In Section 5, we use numerical simulation to illustrate our results.

\section{Existence and Uniqueness of Global Positive Solution}

First, to facilitate the analysis that follows, we make the following tags. When $f(t)$ is a continuous $T$-periodic function, we define:

$$
f^{u}=\sup _{t \geq 0} f(t), f^{l}=\inf _{t \geq 0} f(t)
$$

Moreover, we assume that a product equals unity if the number of factors is zero.

Definition 2.1. [8] Consider an impulsive stochastic differential equation

$$
\left\{\begin{array}{l}
\mathrm{d} x(t)=f(t, x(t)) \mathrm{d} t+g(t, x(t)) \mathrm{d} B(t), t \neq t_{k}, t>0, \\
x\left(t_{k}^{+}\right)-x\left(t_{k}\right)=\alpha_{k} x\left(t_{k}\right), t=t_{k}, k=1,2,3, \cdots
\end{array}\right.
$$

A stochastic process $x(t)=\left(x_{1}(t), x_{2}(t), \cdots, x_{n}(t)\right)^{\mathrm{T}}, t \in[0,+\infty)$ is said to be a solution of ISDE (2.1), if $x(t)$ satisfies

1) $x(t)$ is $\mathcal{F}_{t}$ adapted and is continuous on $\left(0, t_{1}\right)$ and each interval $\left(t_{k}, t_{k+1}\right), k \in \mathbb{N}$ and $f(t, x(t)) \in L^{1}\left(\mathbb{R}^{+}, \mathbb{R}^{n}\right), g(t, x(t)) \in L^{2}\left(\mathbb{R}^{+}, \mathbb{R}^{n}\right)$;

2) $x(t)$ obeys the equivalent integral equation of (2.1) for almost every $t \in \mathbb{R}_{+} \backslash t_{k}$ and satisfies the impulsive conditions at each $t \in \mathbb{R}_{+}, k \in \mathbb{N}$ a.s.;

3) For each $t_{k}, k \in \mathbb{N}, x\left(t_{k}^{+}\right)=\lim _{t \rightarrow t_{k}^{+}} x(t)$ and $\left.x\left(t_{k}^{-}\right)\right)=\lim _{t \rightarrow t_{k}^{-}} x((t))$ exist and $x\left(t_{k}^{-}\right)=x\left(t_{k}\right)$ with probability one.

We give the main results of system (1.3) as follows.

Theorem 2.1. For any initial value $\left(x_{0}, y_{0}\right) \in R_{+}^{2}$ the system (1.3) has a unique global positive solution $(x(t), y(t))$ for $t \geq 0$ and the solution remains in $\mathbb{R}_{+}$with probability one.

Proof. First, we construct the following SDE without impulses:

$$
\left\{\begin{aligned}
\mathrm{d} x_{1}(t)= & x_{1}(t)\left[r_{1}(t)+\frac{1}{T} \sum_{j=1}^{p} \ln \left(1+\alpha_{j}\right)-a_{11}(t) A_{1}(t) x_{1}(t)-a_{12}(t) A_{2}(t) x_{2}(t)\right. \\
& \left.-\frac{H(t)}{1+b(t) A_{1}(t) x_{1}(t)}\right] \mathrm{d} t+\sigma_{1}(t) x_{1}(t) \mathrm{d} B_{1}(t), \\
\mathrm{d} x_{2}(t)= & x_{2}(t)\left[-r_{2}(t)+\frac{1}{T} \sum_{j=1}^{p} \ln \left(1+\beta_{j}\right)+a_{21}(t) A_{1}(t) x_{1}(t)-a_{22}(t) A_{2}(t) x_{2}(t)\right. \\
& \left.-\frac{h(t)}{1+b(t) A_{2}(t) x_{2}(t)}\right] \mathrm{d} t-\sigma_{2}(t) x_{2}(t) \mathrm{d} B_{2}(t)-\frac{\sigma_{3}(t) h(t) x_{2}(t)}{1+b(t) x_{2}(t)} \mathrm{d} B_{3}(t),
\end{aligned}\right.
$$


with the initial value $\left(x_{1}, x_{2}\right)=\left(x_{0}, y_{0}\right)$, where

$$
A_{1}(t)=\left(\prod_{j=1}^{p}\left(1+\alpha_{j}\right)\right)^{-\frac{t}{T}} \prod_{0 \leq t_{k}<t}\left(1+\alpha_{k}\right), \quad A_{2}(t)=\left(\prod_{j=1}^{p}\left(1+\beta_{j}\right)\right)^{-\frac{t}{T}} \prod_{0 \leq t_{k}<t}\left(1+\beta_{k}\right)
$$

Then it is obvious that $A_{1}(t), A_{2}(t)$ are positive $T$-periodic functions. In fact,

$$
\begin{aligned}
\frac{A_{1}(t+T)}{A_{2}(t)} & =\frac{\left(\prod_{j=1}^{p}\left(1+\alpha_{j}\right)\right)^{-\frac{t+T}{T}} \prod_{0 \leq t_{k}<t+T}\left(1+\alpha_{k}\right)}{\left(\prod_{j=1}^{p}\left(1+\alpha_{j}\right)\right)^{-\frac{t}{T}} \prod_{0 \leq t_{k}<t}\left(1+\alpha_{k}\right)} \\
& =\left(\prod_{j=1}^{p}\left(1+\alpha_{j}\right)\right)^{-1} \prod_{t \leq t_{k}<t+T}\left(1+\alpha_{k}\right) .
\end{aligned}
$$

For any $t \geq 0$, there is an integer $n$, such that

$$
n T \leq t \leq(n+1) T .
$$

The limited mathematical induction procedures, together with $t_{k+p}=t_{k}+T$, $\alpha_{k+p}=\alpha_{k}$ induce that

$$
t_{k+n p}=t_{k+(n-1) p}+T=\cdots=t_{k}+n T, \alpha_{k+n p}=\alpha_{k+(n-1) p}=\cdots=\alpha_{k}
$$

According to $[0, T) \cap\left\{t_{k}, k \in \mathbb{Z}\right\}=\left\{t_{1}, t_{2}, \cdots, t_{p}\right\}$, there exists $l=\{1,2, \cdots, p\}$ such that

$$
\begin{aligned}
& t_{l+n p}, t_{l+1+n p}, \cdots, t_{p+n p} \in[t,(n+1) T), \\
& t_{1+(n+1) p}, t_{2+(n+1) p}, \cdots, t_{l-1+(n+1) p} \in[(n+1) T, t+T) .
\end{aligned}
$$

Thus, combining (2.2)-(2.4), we obtain

$$
\begin{aligned}
A_{1}(t+T) & =A_{1}(t)\left(\prod_{j=1}^{p}\left(1+\alpha_{j}\right)\right)^{-1} \prod_{k=1}^{p}\left(1+\alpha_{k+n p}\right) \prod_{k=1}^{l-1}\left(1+\alpha_{k+(n+1) p}\right) \\
& =A_{1}(t)\left(\prod_{j=1}^{p}\left(1+\alpha_{j}\right)\right)^{-1} \prod_{k=l}^{p}\left(1+\alpha_{k+n p}\right) \prod_{k=1}^{l-1}\left(1+\alpha_{k}\right) \\
& =A_{1}(t)\left(\prod_{j=1}^{p}\left(1+\alpha_{j}\right)\right)^{-1} \prod_{k=1}^{p}\left(1+\alpha_{k}\right) \\
& =A_{1}(t),
\end{aligned}
$$

Similarly, $A_{2}(t+T)=A_{2}(t)$.

By the same method as [9] and standard proof [10], Equation (2.2) has a unique global positive Solution $\left(x_{1}(t), x_{2}(t)\right)$.

Next we will show that $(x(t), y(t))$ is the solution of system (2.2), which is continuous on each interval $\left(t_{k}, t_{k+1}\right) \in R^{+}$. For any $t \neq t_{k}$.

Let

$$
\begin{aligned}
& x(t)=A_{1}(t) x_{1}(t), \quad y(t)=A_{2}(t) x_{2}(t) . \\
\mathrm{d} x(t)= & A_{1}^{\prime}(t) x_{1}(t) \mathrm{d} t+A_{1}(t) \mathrm{d} x_{1}(t) \\
= & x(t)\left[r_{1}(t)-a_{11}(t) x(t)-a_{12}(t) y(t)-\frac{H(t)}{1+b(t) x(t)}\right] \\
& +\sigma_{1}(t) x_{1}(t) \mathrm{d} w_{1}(t),
\end{aligned}
$$




$$
\begin{aligned}
\mathrm{d} y(t)= & y(t)\left[-r_{2}(t)+a_{21}(t) x(t)-a_{22}(t) y(t)-\frac{h(t)}{1+b(t) y(t)}\right] \mathrm{d} t \\
& -\sigma_{2}(t) y(t) \mathrm{d} w_{2}(t)-\frac{\sigma_{3}(t) h(t) y(t)}{1+b(t) y(t)} \mathrm{d} w_{3}(t)
\end{aligned}
$$

And, for every $k \in N$,

$$
\begin{gathered}
x\left(t_{k}^{+}\right)=\lim _{t \rightarrow t_{k}^{+}} A_{1}(t) x_{1}(t)=\left(\prod_{j=1}^{p}\left(1+\alpha_{j}\right)\right)^{-\frac{t_{k}}{\gamma}} \prod_{0 \leq t_{k}<t_{k}}\left(1+\alpha_{k}\right) x_{1}\left(t_{k}\right)=\left(1+\alpha_{j}\right) x\left(t_{k}\right), \\
x\left(t_{k}^{-}\right)=\lim _{t \rightarrow t_{\bar{k}}^{-}} A_{1}(t) x_{1}(t)=A_{1}\left(t_{k}\right) x_{1}\left(t_{k}^{-}\right)=A_{1}\left(t_{k}\right) x_{1}\left(t_{k}\right)=x\left(t_{k}\right) .
\end{gathered}
$$

Similarly, we can show that,

$$
y\left(t_{k}^{+}\right)=\left(1+\beta_{j}\right) y\left(t_{k}\right), y\left(t_{k}^{-}\right)=y\left(t_{k}\right) .
$$

Therefore, $(x(t), y(t))$ is a solution that satisfies system (1.3) Finally, we prove the nonnegative uniqueness of the solution of system (1.3) (more details see [11]).

Then the proof is completed.

\section{Existence of Periodic Solutions of the System}

In this section, we give the existence of the positive periodic solution of the stochastic system (1.3) with impulses. For convenience of readers, we first give the definition of the periodic solution of the impulsive stochastic differential equation in the sense of distribution and the results of the existence of periodic solutions (see [10] [11]).

Definition 3.1. [12] A stochastic process $\xi(t)=\xi(t, w)$ is said to be periodic with period $T$, if for every finite sequence of numbers $t_{1}, t_{2}, \cdots, t_{n}$ the joint distribution of random variables $\xi\left(t_{1}+h\right), \xi\left(t_{2}+h\right), \cdots, \xi\left(t_{n}+h\right)$ is independent of $h$, where $h=k T(k= \pm 1, \pm 2, \cdots)$.

Consider the following periodic stochastic differential equation without impulse:

$$
\mathrm{d} x(t)=f(t, x(t)) \mathrm{d} t+g(t, x(t)) \mathrm{d} B(t), t \geq 0
$$

where $g(t, x(t))_{n \times l}$ is a $n \times l$ matrix function, $f(t, x(t))$ and the matrix $g(t, x(t))_{n \times l}$ are $T$-periodic in $t$.

Lemma 3.1. [12] [13] Assume that the system (3.1) has a global solution, and there exists a $T$-periodic function $V(t, x)$ such that the following conditions hold:

1) $L V(t, x) \leq-1$ on the outside of some compact set;

2) $\inf _{|x|>R} \rightarrow \infty$, as $R \rightarrow \infty$.

Then Equation (3.1) has a $T$-periodic solution.

According to Lemma 3.2, we can obtain the main result in this section.

Theorem 3.1. Assume 


$$
\begin{aligned}
& \lambda_{1}=\frac{1}{T} \int_{0}^{T}\left(a_{21}^{l} A_{1}^{u}\left(r_{1}(t)+\frac{1}{T} \sum_{j=1}^{p} \ln \left(1+\alpha_{j}\right)-H(t)-\frac{\sigma_{1}^{2}(t)}{2}\right)\right. \\
& \left.(H 1): \quad-a_{11}^{u} A_{1}^{l}\left(-r_{2}(t)+\frac{1}{T} \sum_{j=1}^{p} \ln \left(1+\beta_{j}\right)-h(t)-\frac{\sigma_{2}^{2}(t)}{2}-\frac{\sigma_{3}^{2}(t) h^{2}(t)}{2}\right)\right) \mathrm{d} t \\
& >0 \text {, } \\
& \text { (H2): } \lambda_{2}=\frac{1}{T} \int_{0}^{T}\left(r_{2}(t)-\frac{1}{T} \sum_{j=1}^{p} \ln \left(1+\beta_{j}\right)-\frac{\sigma_{2}^{2}(t)}{2}-\frac{\sigma_{3}^{2}(t) h^{2}(t)}{2}\right) \mathrm{d} t>0, \\
& \text { (H3): } \frac{a_{21}^{l} A_{1}^{u}}{A_{1}^{l}} a_{11}^{u} A_{1}^{u} \leq a_{11}^{u} A_{1}^{l} a_{21}^{l} A_{1}^{l} \text {, } \\
& \text { (H4): } \frac{C A_{2}^{u}\left(a_{21}^{l} a_{12}^{u} A_{1}^{u}+a_{11}^{u} a_{22}^{u}\right)}{r_{2}^{l} A_{1}^{l} T} \sum_{j=1}^{p} \ln \left(1+\beta_{j}\right) \leq \frac{C A_{2}^{u}\left(a_{21}^{l} a_{12}^{u} A_{1}^{u}+a_{11}^{u} a_{22}^{u}\right)}{A_{1}^{l}} .
\end{aligned}
$$

Then system (1.4) has a positive $T$-periodic solution.

Proof. We only need to prove the existence of a periodic solution of the equivalent system (2.2) without impulses. The global existence of the solution has been ensured by Theorem 1 . Then, we only have to verify the conditions of by Lemma 3.1.

Define a $C^{2}$-function $V(t, x, y): \mathbb{R}_{+}^{2} \rightarrow \mathbb{R}_{+}$:

$$
\begin{aligned}
V(t, x, y)= & C\left(-\frac{a_{21}^{l} A_{1}^{u}}{A_{1}^{l}} \ln x-a_{11}^{u} A_{1}^{u} \ln y+w_{1}(t)+\frac{A_{2}^{u}\left(a_{21}^{l} a_{12}^{u} A_{1}^{u}+a_{11}^{u} a_{22}^{u}\right)}{r_{2}^{l} A_{1}^{l}} y\right) \\
& +\mathrm{e}^{w_{2}(t)} \frac{\left(\frac{a_{21}^{u}}{a_{12}^{l}} A_{1}^{u} x+A_{2}^{l}\right)^{2} y}{2\left(A_{2}^{l}\right)^{2}} \\
\triangleq & V_{1}(t, x, y)+V_{2}(t, x, y) .
\end{aligned}
$$

where $C>0$ will be determined later. Here, $w_{i}(t)(i=1,2)$ satisfies

$$
\begin{gathered}
w_{1}^{\prime}(t)=a_{21}^{l} A_{1}^{u}\left(r_{1}(t)-H(t)-\frac{\sigma_{1}^{2}(t)}{2}\right)-a_{11}^{u} A_{1}^{l}\left(-r_{2}(t)-\frac{\sigma_{2}^{2}(t)+\sigma_{3}^{2}(t) h^{2}(t)}{2}\right) \\
-\left\langle a_{21}^{l} A_{1}^{u}\left(r_{1}(t)-H(t)-\frac{\sigma_{1}^{2}(t)}{2}\right)-a_{11}^{u} A_{1}^{l}\left(-r_{2}(t)-\frac{\sigma_{2}^{2}(t)+\sigma_{3}^{2}(t) h^{2}(t)}{2}\right)\right\rangle_{T} \\
w_{2}^{\prime}(t)=2 r_{2}(t)-\sigma_{2}^{2}(t)-\sigma_{3}^{2}(t) h^{2}(t)-\left\langle 2 r_{2}(t)-\sigma_{2}^{2}(t)-\sigma_{3}^{2}(t) h^{2}(t)\right\rangle_{T}
\end{gathered}
$$

Which $\lambda_{1}$ and $\lambda_{2}$ are defined by (H1), (H2). Obviously, $w_{i}(t)$ are $T$-periodic functions. And $w_{2}^{\prime}(t)$ is a bounded function. Thus there is $K>0$ such that;

$$
\left|w_{2}^{\prime}(t)\right| \leq K, \quad \forall t \geq 0
$$

In order to confirm the condition (2) of Lemma 3.1, we only need to prove that

$$
\inf _{(t . x . y) \in[0, \infty) \times\left(R_{+}^{2} \backslash U_{k}\right)} V(t, x, y) \rightarrow \infty \text {, as } k \rightarrow \infty .
$$


where $U_{k}=\left(\frac{1}{k}, k\right) \times\left(\frac{1}{k}, k\right)$, here the coefficients of the quadratic term $x^{2}, y^{2}$ of $V(t, x, y)$ are all positive.

Next, we verify the condition (1) of Lemma 3.2. By Itô's formula, we have:

$$
\begin{aligned}
& L V_{1} \leq \frac{C a_{21}^{l} A_{1}^{u}}{A_{1}^{l}}\left(-r_{1}(t)-\frac{1}{T} \sum_{j=1}^{p} \ln \left(1+\alpha_{j}\right)+\frac{\sigma_{1}^{2}(t)}{2}+a_{11}(t) A_{1}(t) x\right. \\
& \left.+a_{12}(t) A_{2}(t) y+H(t)\right)+C w_{1}^{\prime}(t)+C a_{11}^{u} A_{1}^{l}\left(r_{2}(t)-\frac{1}{T} \sum_{j=1}^{p} \ln \left(1+\beta_{j}\right)\right. \\
& \left.-a_{21} A_{1}(t) x+a_{22}(t) A_{2}(t) y+h(t) \frac{\sigma_{2}^{2}(t)+\sigma_{3}^{2}(t)}{2}\left(\frac{h(t)}{1+b(t) A_{2}(t) y}\right)^{2}\right) \\
& +\frac{C A_{2}^{u}\left(a_{21}^{l} a_{12}^{u} A_{1}^{u}+a_{11}^{u} a_{22}^{u}\right)}{r_{2}^{l} A_{1}^{l}} y\left(-r_{2}(t)+\frac{1}{T} \sum_{j=1}^{p} \ln \left(1+\beta_{j}\right)+a_{21}(t) A_{1}(t) x\right. \\
& \left.-a_{22}(t) A_{2}(t) y-\frac{h(t)}{1+b(t) A_{2}(t) y}\right) \\
& \leq \frac{C a_{21}^{l} A_{1}^{u}}{A_{1}^{l}}\left(-r_{1}(t)+C w_{1}^{\prime}(t)+\frac{1}{T} \sum_{j=1}^{p} \ln \left(1+\alpha_{j}\right)+H(t)+\frac{\sigma_{1}^{2}(t)}{2}\right) \\
& +C a_{11}^{u} A_{1}^{l}\left(r_{2}(t)-\frac{1}{T} \sum_{j=1}^{p} \ln \left(1+\beta_{j}\right)+h(t)+\frac{\sigma_{2}^{2}(t)+\sigma_{3}^{2}(t) h(t)^{2}}{2}\right) \\
& +C w_{1}^{\prime}(t)+\frac{C a_{21}^{u} A_{1}^{u} A_{2}^{u}\left(a_{21}^{l} a_{12}^{u} A_{1}^{u}+a_{11}^{u} a_{22}^{u}\right)}{r_{2}^{l} A_{1}^{l}} x y \\
& +\left(\frac{C a_{21}^{l} A_{1}^{u}}{A_{1}^{l}} a_{11}(t) A_{1}(t)-C a_{11}^{u} A_{1}^{l} a_{21}(t) A_{1}(t)\right) x \\
& +\left(\frac{C a_{21}^{l} A_{1}^{u}}{A_{1}^{l}} a_{12}(t) A_{2}(t)+\frac{C A_{2}^{u}\left(a_{21}^{l} a_{12}^{u} A_{1}^{u}+a_{11}^{u} a_{22}^{u}\right)}{r_{2}^{l} A_{1}^{l} T} \sum_{j=1}^{p} \ln \left(1+\beta_{j}\right)\right. \\
& \left.-\frac{C A_{2}^{u}\left(a_{21}^{l} a_{12}^{u} A_{1}^{u}+a_{11}^{u} a_{22}^{u}\right)}{A_{1}^{l}}\right) y \\
& \leq C\left(-\lambda_{1}+\frac{a_{21}^{u} A_{1}^{u} A_{2}^{u}\left(a_{21}^{l} a_{12}^{u} A_{1}^{u}+a_{11}^{u} a_{22}^{u}\right)}{r_{2}^{l} A_{1}^{l}} x y+\frac{a_{21}^{l} A_{1}^{u}}{A_{1}^{l}} a_{12}(t) A_{2}(t) y\right) . \\
& \text { As } V_{2}(t, x, y)=\mathrm{e}^{w_{2}(t)} \frac{\left(\frac{a_{21}^{u}}{a_{12}^{l}} A_{1}^{u} x(t)+A_{2}^{l}\right)^{2}}{2\left(A_{2}^{l}\right)^{2}} y \text { so that: } \\
& V_{2}(t, x, y)=\frac{1}{2} \mathrm{e}^{w_{2}(t)}\left(\frac{a_{21}^{u} A_{1}^{u}}{a_{12}^{l} A_{2}^{l}} x\right)^{2}+\frac{a_{21}^{u} A_{1}^{u}}{a_{12}^{l} A_{2}^{l}} \mathrm{e}^{w_{2}(t)} x y+\frac{1}{2} \mathrm{e}^{w_{2}(t)} y^{2} \\
& \text { Let } V_{3}(t, x, y)=\frac{1}{2} \mathrm{e}^{w_{2}(t)}\left(\frac{a_{21}^{u} A_{1}^{u}}{a_{12}^{l} A_{2}^{l}} x\right)^{2}, V_{4}(t, x, y)=\frac{a_{21}^{u} A_{1}^{u}}{a_{12}^{l} A_{2}^{l}} \mathrm{e}^{w_{2}(t)} x y \text {, } \\
& V_{5}(t, x, y)=\frac{1}{2} \mathrm{e}^{w_{2}(t)} y^{2} \text {, we have }
\end{aligned}
$$




$$
\begin{aligned}
& L V_{3}(t, x, y) \\
& =\mathrm{e}^{w_{2}(t)}\left(\frac{a_{21}^{u} A_{1}^{u}}{a_{12}^{l} A_{2}^{l}}\right)^{2}\left[x^{2}\left(\frac{w_{2}^{\prime}(t)}{2}+r_{1}(t)+\frac{1}{T} \sum_{j=1}^{p} \ln \left(1+\alpha_{j}\right)+\frac{\sigma_{1}^{2}(t)}{2}\right)\right. \\
& \left.+\left(-a_{11}(t) A_{1}(t) x^{3}-a_{12}(t) A_{2}(t) x^{2} y-\frac{H(t) x^{2}}{1+b(t) A_{1}(t) x}\right)\right] \\
& \leq \mathrm{e}^{w_{2}(t)}\left(\frac{a_{21}^{u} A_{1}^{u}}{a_{12}^{l} A_{2}^{l}}\right)^{2}\left[x^{2}\left(\frac{K}{2}+r_{1}^{u}+\frac{1}{T} \sum_{j=1}^{p} \ln \left(1+\alpha_{j}\right)+\frac{\left(\sigma_{1}^{2}\right)^{u}}{2}\right)-a_{11}^{l} A_{1}^{l} x^{3}-a_{12}^{l} A_{2}^{l} x^{2} y\right] \text {, } \\
& L V_{4}(t, x, y) \\
& =\mathrm{e}^{w_{2}(t)} \frac{a_{21}^{u} A_{1}^{u}}{a_{12}^{l} A_{2}^{l}}\left[w_{2}^{\prime}(t)+r_{1}(t)+\frac{1}{T} \sum_{j=1}^{p} \ln \left(1+\alpha_{j}\right)-r_{2}(t)+\frac{1}{T} \sum_{j=1}^{p} \ln \left(1+\beta_{j}\right)\right. \\
& -\left(a_{11}(t) A_{1}(t) x^{2} y+a_{12}(t) A_{2}(t) y^{2} x-a_{21}(t) A_{1}(t) x^{2} y+a_{22}(t) A_{2}(t) y^{2} x\right) \\
& \left.-\left(\frac{H(t)}{1+b(t) A_{1}(t) x}+\frac{h(t)}{1+b(t) A_{2}(t) y}\right) x y\right] \\
& \leq \mathrm{e}^{w_{2}(t)} \frac{a_{21}^{u} A_{1}^{u}}{a_{12}^{l} A_{2}^{l}}\left[K+r_{1}^{u}+\frac{1}{T} \sum_{j=1}^{p} \ln \left(1+\alpha_{j}\right)+r_{2}^{u}\right. \\
& \left.+\frac{1}{T} \sum_{j=1}^{p} \ln \left(1+\beta_{j}\right)+a_{21}^{u} A_{1}^{u} x^{2} y-a_{12}^{l} A_{2}^{l} y^{2} x\right] \text {, } \\
& L V_{5}(t, x, y) \\
& =\mathrm{e}^{w_{2}(t)}\left(\frac{w_{2}^{\prime}(t)}{2}-r_{2}(t)+\frac{1}{T} \sum_{j=1}^{p} \ln \left(1+\beta_{j}\right)+\frac{\sigma_{2}^{2}(t)}{2}+\frac{\sigma_{3}^{2}(t) h^{2}(t)}{2}\right) y^{2} \\
& +\mathrm{e}^{w_{2}(t)}\left(-a_{22}(t) A_{2}(t) y^{3}+a_{21}(t) A_{1}(t) y^{2} x-\frac{h(t) y^{2}}{1+b(t) A_{2}(t) y}\right) \\
& \leq-\mathrm{e}^{w_{2}(t)} \lambda_{2} y^{2}+\mathrm{e}^{w_{2}(t)}\left(-a_{22}(t) A_{2}(t) y^{3}+a_{21}^{u} A_{1}^{u} y^{2} x\right) .
\end{aligned}
$$

Then

$$
\begin{aligned}
& L V_{2}(t, x, y) \\
& \leq \mathrm{e}^{\left|w_{2}^{u}\right|}\left(\frac{a_{21}^{u} A_{1}^{u}}{a_{12}^{l} A_{2}^{l}}\right)^{2} x^{2}\left[\frac{K}{2}+r_{1}^{u}+\frac{1}{T} \sum_{j=1}^{p} \ln \left(1+\alpha_{j}\right)+\frac{\left(\sigma_{1}^{2}\right)^{u}}{2}\right] \\
& +\frac{a_{21}^{u} A_{1}^{u}}{a_{12}^{l} A_{2}^{l}} \mathrm{e}^{\left|w_{2}^{u}\right|} x y\left[K+r_{1}^{u}+\frac{1}{T} \sum_{j=1}^{p} \ln \left(1+\alpha_{j}\right)+r_{2}^{u}+\frac{1}{T} \sum_{j=1}^{p} \ln \left(1+\beta_{j}\right)\right] \\
& -\mathrm{e}^{w_{2}^{l}}\left(\left(\frac{a_{21}^{u} A_{1}^{u}}{a_{12}^{l} A_{2}^{l}}\right)^{2} a_{11}^{l} A_{1}^{l} x^{3}+a_{22}^{l} A_{2}^{l} y^{3}+\lambda_{2} y^{2}\right) .
\end{aligned}
$$

Then

$$
\begin{aligned}
& L V(t, x, y) \\
& =L V_{1}(t, x, y)+L V_{2}(t, x, y) \\
& =-C \lambda_{1}+m_{1} x y+m_{2} x^{2}-\mathrm{e}^{w_{2}^{l}}\left(\left(\frac{a_{21}^{u} A_{1}^{u}}{a_{12}^{l} A_{2}^{l}}\right)^{2} a_{11}^{l} A_{1}^{l} x^{3}+a_{22}^{l} A_{2}^{l} y^{3}+\lambda_{2} y^{2}\right) .
\end{aligned}
$$


where:

$$
\begin{aligned}
m_{1}= & \frac{C a_{21}^{u} A_{1}^{u} A_{2}^{u}\left(a_{21}^{l} a_{12}^{u} A_{1}^{u}+a_{11}^{u} a_{22}^{u}\right)}{r_{2}^{l} A_{1}^{l}} \\
& +\frac{a_{21}^{u} A_{1}^{u} \mathrm{e}^{\left|w_{2}^{u}\right|}}{a_{12}^{l} A_{2}^{l}}\left[K+r_{1}^{u}+\frac{1}{T} \sum_{j=1}^{p} \ln \left(1+\alpha_{j}\right)+r_{2}^{u}+\frac{1}{T} \sum_{j=1}^{p} \ln \left(1+\beta_{j}\right)\right], \\
& m_{2}=\mathrm{e}^{\left|w_{2}^{u}\right|}\left(\frac{a_{21}^{u} A_{1}^{u}}{a_{12}^{l} A_{2}^{l}}\right)^{2}\left[\frac{K}{2}+r_{1}^{u}+\frac{1}{T} \sum_{j=1}^{p} \ln \left(1+\alpha_{j}\right)+\frac{\left(\sigma_{1}^{2}\right)^{u}}{2}\right]
\end{aligned}
$$

Let, we take

$$
C=\frac{2}{\lambda_{1}} \max \left\{2, m_{2} x^{2}-\frac{\mathrm{e}^{w_{2}^{l}}}{2}\left(\frac{a_{21}^{u} A_{1}^{u}}{a_{12}^{l} A_{2}^{l}}\right)^{2} a_{11}^{l} A_{1}^{l} x^{3}-\frac{\mathrm{e}^{w_{2}^{l}}}{2} a_{22}^{l} A_{2}^{l} y^{3}-\lambda_{2} y^{2}\right\}
$$

To confirm the condition (1) of Lemma 3.2, we choose a sufficiently small constant $\varepsilon$ such that:

$$
\begin{gathered}
0<\varepsilon \leq\left\{\frac{C \lambda_{1}}{4 m_{1}}, \frac{\mathrm{e}^{w_{2}^{l}}\left(a_{21}^{u} A_{1}^{u}\right)^{2} a_{11}^{l} A_{1}^{l}}{2 m_{1}\left(a_{12}^{l} A_{2}^{l}\right)^{2}}, \frac{\mathrm{e}^{w_{2}^{l}} a_{22}^{l} A_{2}^{l}}{2 m_{1}}\right\} \\
-C \lambda_{1}+C_{1}+1 \leq \mathrm{e}^{w_{2}^{l}} \min \left\{\frac{\left(a_{21}^{u} A_{1}^{u}\right)^{2} a_{11}^{l} A_{1}^{l}}{2\left(a_{12}^{l} A_{2}^{l}\right)^{2} \varepsilon^{3}}, \frac{a_{22}^{l} A_{2}^{l}}{2 \varepsilon^{3}}\right\}
\end{gathered}
$$

where

$$
\begin{aligned}
C_{1}= & \max _{(x, y) \in \mathbb{R}_{+}^{2}}\left\{\frac{2}{5} m_{1} x^{\frac{5}{2}}+\frac{3}{5} m_{1} y^{\frac{5}{3}}+m_{2} x^{2}\right. \\
& \left.-\frac{\mathrm{e}^{w_{2}^{l}}}{2}\left(\left(\frac{a_{21}^{u} A_{1}^{u}}{a_{12}^{l} A_{2}^{l}}\right)^{2} a_{11}^{l} A_{1}^{l} x^{3}+a_{22}^{l} A_{2}^{l} y^{3}\right)+\mathrm{e}^{w_{2}^{l}} \lambda_{2} y^{2}\right\}
\end{aligned}
$$

Define a bounded open set

$$
D_{\varepsilon}=\left\{(x, y) \mid \varepsilon<x<\frac{1}{\varepsilon}, \varepsilon<y<\frac{1}{\varepsilon}\right\} .
$$

and denote

$$
\begin{array}{cc}
D_{\varepsilon}^{1}=\{(x, y) \mid 0<x \leq \varepsilon\}, & D_{\varepsilon}^{2}=\{(x, y) \mid 0<y \leq \varepsilon\}, \\
D_{\varepsilon}^{3}=\left\{(x, y) \mid x \geq \frac{1}{\varepsilon}\right\}, & D_{\varepsilon}^{4}=\left\{(x, y) \mid y \geq \frac{1}{\varepsilon}\right\} .
\end{array}
$$

It is obvious that $D_{\varepsilon}^{c}=D_{\varepsilon}^{1} \cup D_{\varepsilon}^{2} \cup D_{\varepsilon}^{3} \cup D_{\varepsilon}^{4}$. Next, $L V(t, x, y) \leq-1$ on $[0, \infty) \times D_{\varepsilon}^{c}$ must be shown.

Case 1: If $(t, x, y) \in D_{\varepsilon}^{1}$, then $x y \leq \varepsilon y \leq \varepsilon\left(1+y^{3}\right)$, we have:

$$
\begin{aligned}
& L V(t, x, y) \\
& \leq-\frac{C \lambda_{1}}{4}+\left(-\frac{C \lambda_{1}}{4}+m_{1} \varepsilon\right)-\frac{\mathrm{e}^{w_{2}^{l}}}{2}\left(\frac{a_{21}^{u} A_{1}^{u}}{a_{12}^{l} A_{2}^{l}}\right)^{2} a_{11}^{l} A_{1}^{l} x^{3}-\left(\frac{e^{w_{2}^{l}}}{2} a_{22}^{l} A_{2}^{l}-\varepsilon\right) y^{3} \\
& +\left\{-\frac{C \lambda_{1}}{2}+m_{2} x^{2}-\frac{\mathrm{e}^{w_{2}^{l}}}{2}\left(\frac{a_{21}^{u} A_{1}^{u}}{a_{12}^{l} A_{2}^{l}}\right)^{2} a_{11}^{l} A_{1}^{l} x^{3}-\frac{\mathrm{e}^{w_{2}^{l}}}{2} a_{22}^{l} A_{2}^{l} y^{3}-\lambda_{2} y^{2}\right\},
\end{aligned}
$$


Using (3.8) and (3.9), we obtain

$$
L V(t, x, y) \leq-\frac{C \lambda_{1}}{4}-\frac{\mathrm{e}^{w_{2}^{l}}}{2}\left(\frac{a_{21}^{u} A_{1}^{u}}{a_{12}^{l} A_{2}^{l}}\right)^{2} a_{11}^{l} A_{1}^{l} x^{3} \leq-\frac{C \lambda_{1}}{4} \leq-1 .
$$

Case 2: If $(t, x, y) \in D_{\varepsilon}^{2}$, then $x y \leq \varepsilon x \leq \varepsilon\left(1+x^{3}\right)$, we have:

$$
\begin{aligned}
& L V(t, x, y) \\
& \leq-\frac{C \lambda_{1}}{4}+\left(-\frac{C \lambda_{1}}{4}+m_{1} \varepsilon\right)-\left(\frac{\mathrm{e}^{w_{2}^{l}}}{2}\left(\frac{a_{21}^{u} A_{1}^{u}}{a_{12}^{l} A_{2}^{l}}\right)^{2} a_{11}^{l} A_{1}^{l}-\varepsilon\right) x^{3}-\frac{\mathrm{e}^{w_{2}^{l}}}{2} a_{22}^{l} A_{2}^{l} y^{3} \\
& +\left\{-\frac{C \lambda_{1}}{2}+m_{2} x^{2}-\frac{\mathrm{e}^{w_{2}^{l}}}{2}\left(\frac{a_{21}^{u} A_{1}^{u}}{a_{12}^{l} A_{2}^{l}}\right)^{2} a_{11}^{l} A_{1}^{l} x^{3}-\frac{\mathrm{e}^{w_{2}^{l}}}{2} a_{22}^{l} A_{2}^{l} y^{3}-\lambda_{2} y^{2}\right\},
\end{aligned}
$$

By the definition (3.8) of $\mathrm{C}$ and the inequalities (3.9), we have:

$$
L V(t, x, y) \leq-\frac{C \lambda_{1}}{4}-\frac{\mathrm{e}^{w_{2}^{l}}}{2} a_{22}^{l} A_{2}^{l} y^{3} \leq-\frac{C \lambda_{1}}{4} \leq-1 .
$$

By Young inequality, we have $x y \leq \frac{2}{5} x^{\frac{5}{2}}+\frac{3}{5} y^{\frac{5}{3}}$. Then by equality (3.11), the following inequality is obvious:

$$
\begin{aligned}
& L V(t, x, y) \\
& \leq-C \lambda_{1}+m_{2} x^{2}-\mathrm{e}^{w_{2}^{l}}\left(\left(\frac{a_{21}^{u} A_{1}^{u}}{a_{12}^{l} A_{2}^{l}}\right)^{2} a_{11}^{l} A_{1}^{l} x^{3}+a_{22}^{l} A_{2}^{l} y^{3}+\lambda_{2} y^{2}\right)+\frac{2}{5} m_{1} x^{\frac{5}{2}}+\frac{3}{5} m_{2} y^{\frac{5}{3}} \\
& \leq-C \lambda_{1}-\frac{\mathrm{e}^{w_{2}^{l}}}{2}\left(\frac{a_{21}^{u} A_{1}^{u}}{a_{12}^{l} A_{2}^{l}}\right)^{2} a_{11}^{l} A_{1}^{l} x^{3}-\frac{\mathrm{e}^{w_{2}^{l}}}{2} a_{22}^{l} A_{2}^{l} y^{3}+C_{1} .
\end{aligned}
$$

Case 3: If $(t, x, y) \in D_{\varepsilon}^{3}$, from (3.9) and (3.10), we obtain

$$
L V(t, x, y) \leq-C \lambda_{1}-\frac{\mathrm{e}^{w_{2}^{l}}}{2} a_{22}^{l} A_{2}^{l} y^{3}+C_{1} \leq-C \lambda_{1} \mathrm{e}^{w_{2}^{l}} \frac{\left(a_{21}^{u} A_{1}^{u}\right)^{2} a_{11}^{l} A_{1}^{l}}{2\left(a_{12}^{l} A_{2}^{l}\right)^{2} \varepsilon^{3}}+C_{1} \leq-1 .
$$

Case 4: If $(t, x, y) \in D_{\varepsilon}^{4}$, from (3.9) and (3.10), we obtain

$$
L V(t, x, y) \leq-C \lambda_{1}--\frac{\mathrm{e}^{w_{2}^{l}}}{2} a_{22}^{l} A_{2}^{l} y^{3}+C_{1} \leq-C \lambda_{1} \mathrm{e}^{w_{2}^{l}} \frac{a_{11}^{l} A_{1}^{l}}{2 \varepsilon^{3}}+C_{1} \leq-1 .
$$

Thus, we obtain $L V(t, x, y) \leq-1$ on $[0, \infty) \times D_{\varepsilon}^{c}$, and the condition (1) of Lemma 3.2 is satisfied. Therefore, by Lemma 3.2, system (1.3) has a positive $T$-periodic solution.

The proof is confirmed.

\section{Globally Attractive}

Theorem 4.1. [14] [15] If system (1.3) satisfies $a_{11}^{l}-a_{21}^{u}-\frac{H^{u}}{b_{l}^{2}}>0$, $a_{22}^{l}-a_{12}^{u}-\frac{h^{u}}{b_{l}^{2}}>0$, then the system (1.3) is globally attractive. 
Proof. Let $x(t)=\left(x_{1}(t), x_{2}(t)\right), y(t)=\left(y_{1}(t), y_{2}(t)\right)$ be two arbitrary solutions of model (1.3) with initial values $x(t)>0, y(t)>0$.

We defined the following Lyapunov function

$$
V(t)=\left|\ln x_{1}(t)-\ln y_{1}(t)\right|+\left|\ln x_{2}(t)-\ln y_{2}(t)\right|
$$

Then by calculating the right differential $D^{+} V(t)$ and employing Ito's formula.

When $t \neq t_{k}$, we have:

$$
\begin{aligned}
& D^{+} V(t)=\operatorname{sign}\left(x_{1}(t)-y_{1}(t)\right) \mathrm{d}\left(\ln x_{1}(t)-\ln y_{1}(t)\right) \\
&+\operatorname{sign}\left(x_{2}(t)-y_{2}(t)\right) \mathrm{d}\left(\ln x_{2}(t)-\ln y_{2}(t)\right) \\
&=\operatorname{sign}\left(x_{1}(t)-y_{1}(t)\right)\left(-a_{11}(t)\left(x_{1}(t)-y_{1}(t)\right)-a_{12}(t)\left(x_{2}(t)-y_{2}(t)\right)\right. \\
&\left.+\frac{H(t)\left(x_{1}(t)-y_{1}(t)\right)}{\left[1+b(t) x_{1}(t)\right]\left[1+b(t) y_{1}(t)\right]}\right) \mathrm{d} t \\
&+\operatorname{sign}\left(x_{2}(t)-y_{2}(t)\right)\left(a_{21}(t)\left(x_{1}(t)-y_{1}(t)\right)-a_{22}(t)\left(x_{2}(t)-y_{2}(t)\right)\right. \\
&\left.+\frac{h(t)\left(x_{2}(t)-y_{2}(t)\right)}{\left[1+b(t) x_{2}(t)\right]\left[1+b(t) y_{2}(t)\right]}\right) \mathrm{d} t \\
&=\left(-a_{11}(t)\left|x_{1}(t)-y_{1}(t)\right|+a_{12}(t)\left|x_{2}(t)-y_{2}(t)\right|\right. \\
&\left.+\frac{H(t)\left|x_{1}(t)-y_{1}(t)\right|}{\left[1+b(t) x_{1}(t)\right]\left[1+b(t) y_{1}(t)\right]}\right) \mathrm{d} t \\
&+\left(a_{21}(t)\left|x_{1}(t)-y_{1}(t)\right|-a_{22}(t)\left|x_{2}(t)-y_{2}(t)\right|\right. \\
&\left.+\frac{h(t)\left|x_{2}(t)-y_{2}(t)\right|}{\left[1+b(t) x_{2}(t)\right]\left[1+b(t) y_{2}(t)\right]}\right) \mathrm{d} t \\
& \leq-\left[\left(a_{11}^{l}-a_{21}^{u}-\frac{H^{u}}{b_{1}^{2}}\right)\left|x_{1}(t)-y_{1}(t)\right|+\left(a_{22}^{l}-a_{12}^{u}-\frac{h^{u}}{b_{1}^{2}}\right)\left|x_{2}(t)-y_{2}(t)\right|\right] \mathrm{d} t, \\
& \leq-\left(a_{11}(t)-a_{21}(t)-\frac{H(t)}{b^{2}(t)}\right)\left|x_{1}(t)-y_{1}(t)\right| \\
&-\left(a_{22}(t)-a_{12}(t)-\frac{h(t)}{b^{2}(t)}\right)\left|x_{2}(t)-y_{2}(t)\right| \mathrm{d} t \\
& {[(k, N)}
\end{aligned}
$$

when $t=t_{k}, k \in N$, we get

$$
\begin{aligned}
V\left(t_{k}^{+}\right)= & \left|\ln x_{1}\left(t_{k}^{+}\right)-\ln y_{1}\left(t_{k}^{+}\right)\right|+\left|\ln x_{2}\left(t_{k}^{+}\right)-\ln y_{2}\left(t_{k}^{+}\right)\right| \\
= & \left|\ln \left(1+\alpha_{k}\right) x_{1}\left(t_{k}\right)-\ln \left(1+\alpha_{k}\right) y_{1}\left(t_{k}\right)\right| \\
& +\left|\ln \left(1+\beta_{k}\right) x_{2}\left(t_{k}\right)-\ln \left(1+\beta_{k}\right) y_{2}\left(t_{k}\right)\right| \\
= & \left|\ln x_{1}\left(t_{k}\right)-\ln y_{1}\left(t_{k}\right)\right|+\left|\ln x_{2}\left(t_{k}\right)-\ln y_{2}\left(t_{k}\right)\right| \\
= & V\left(t_{k}\right) .
\end{aligned}
$$


Integrating both sides and then taking the expectation yields that

$$
\begin{aligned}
V(t) \leq & V(0)-\left(a_{11}^{l}-a_{21}^{u}-\frac{H^{u}}{b_{l}^{2}}\right) \int_{0}^{t}\left|x_{1}(s)-y_{1}(s)\right| \mathrm{d} s \\
& -\left(a_{22}^{l}-a_{12}^{u}-\frac{h^{u}}{b_{l}^{2}}\right) \int_{0}^{t}\left|x_{2}(s)-y_{2}(s)\right| \mathrm{d} s
\end{aligned}
$$

That is

$$
\begin{aligned}
& V(t)+\left(a_{11}^{l}-a_{21}^{u}-\frac{H^{u}}{b_{l}^{2}}\right) \int_{0}^{t}\left|x_{1}(s)-y_{1}(s)\right| \mathrm{d} s \\
& +\left(a_{22}^{l}-a_{12}^{u}-\frac{h^{u}}{b_{l}^{2}}\right) \int_{0}^{t}\left|x_{2}(s)-y_{2}(s)\right| \mathrm{d} s<V(0)<\infty
\end{aligned}
$$

Then, in the view of $V(t)>0$ and $a_{11}^{l}-a_{21}^{u}-\frac{H^{u}}{b_{l}^{2}}>0, a_{22}^{l}-a_{12}^{u}-\frac{h^{u}}{b_{l}^{2}}>0$ that $\lim _{t \rightarrow \infty} V(t)=0$. Thus, it is easy to see from Lemmas 6.1 [15]

$$
\lim _{t \rightarrow \infty}\left|x_{i}(t)-y_{i}(t)\right|=0, i=1,2 \quad \text { a.s. }
$$

The proof is complete.

\section{Computer Simulations}

In this section, we will prove our theoretical results by some examples with the help of the Matlab software [16] and reveal the influence of impulses and the white noise.

Example 1.

Let

$$
\begin{aligned}
& a_{11}=1.1+\cos t, a_{12}=1.2+\sin t, r_{1}=1+\sin t, H=0.3+0.1 \sin t, \\
& a_{21}=0.05+0.1 \sin t, a_{22}=1.1+\sin t, T=2 \pi, r_{2}=0.15+\sin t, \\
& h=1.5+\sin t, b=2+\sin t, \sigma_{1}=0.03+0.1 \sin t, \sigma_{2}=\sigma_{3}=0.02+0.1 \sin t,
\end{aligned}
$$

then

$$
\begin{gathered}
a_{21}^{l} A_{1}^{u}\left(r_{1}(t)+\frac{1}{T} \sum_{j=1}^{p} \ln \left(1+\alpha_{j}\right)-H(t)-\frac{\sigma_{1}^{2}(t)}{2}\right) \\
+a_{11}^{u} A_{1}^{l}\left(r_{2}(t)-\frac{1}{T} \sum_{j=1}^{p} \ln \left(1+\beta_{j}\right)+h(t)+\frac{\sigma_{2}^{2}(t)}{2}+\frac{\sigma_{3}^{2}(t) h^{2}(t)}{2}\right)=3.13>0, \\
r_{2}(t)-\frac{1}{T} \sum_{j=1}^{p} \ln \left(1+\beta_{j}\right)-\frac{\sigma_{2}^{2}(t)}{2}-\frac{\sigma_{3}^{2}(t) h^{2}(t)}{2} \approx 1.65>0 .
\end{gathered}
$$

Thus, the conditions of Theorem 3.1. hold. Then the model (1.3) has a positive $2 \pi$-periodic solution. Figure 1 confirms the results.

Example 2. Set $a_{11}^{l}=1.1, a_{21}^{u}=0.05, a_{22}^{l}=1.2, a_{12}^{u}=1$. Making condition of the Theorem 4.1 is satisfied. We get that system (1.3) is globally attractive (see Figure 2). 

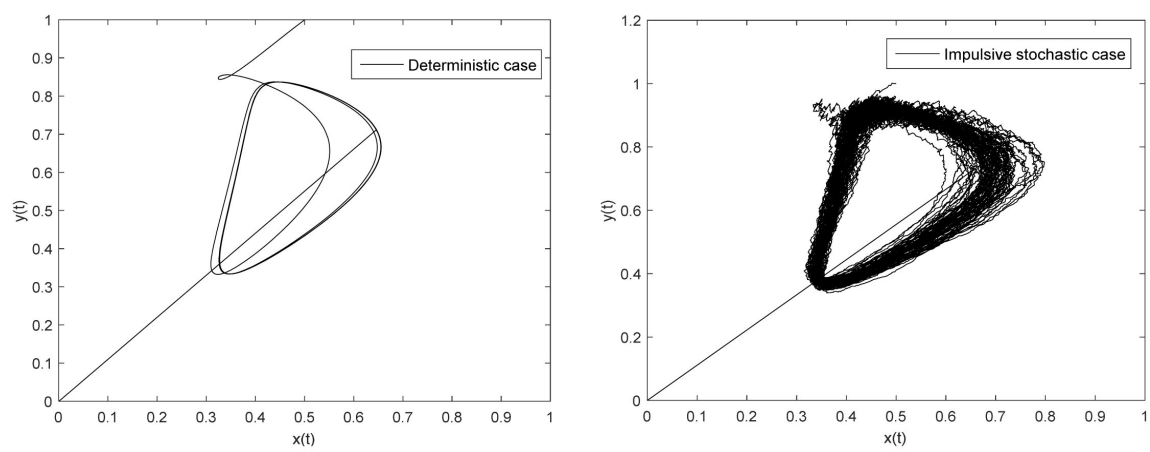

Figure 1. A solution of system (1.4) with the initial value $\left(x_{0}, y_{0}\right)=(0.6,0.3)$. The left is the phase diagram of the stochastic system, and the right is the phase diagram of the deterministic system.
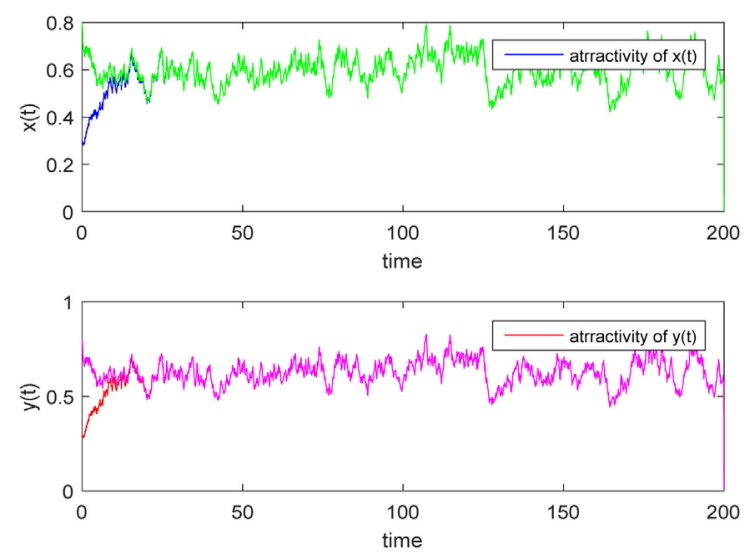

Figure 2. The figure shows the attractiveness of system (1.4), the blue and red lines represent the solution of prey and predator species.

\section{Conclusion}

In this paper, we propose a stochastic predator-prey system with nonlinear harvesting and impulsive perturbations. Firstly, we show that there is a unique positive solution in system (1.3). Secondly, the system has a positive periodic solution under a certain condition. Result shows that when the impulses are sufficiently large such that $\lambda_{1}>0, \lambda_{2}>0$ then the predator and prey will tend to exhibit periodicity. It is verified by constructing the appropriate Lyapunov functions and using Itô's formula. Moreover, these methods used in this study can be extended to more complex and realistic models.

\section{Acknowledgements}

This work was supported by the National Natural Science Foundation of China (11861027) and Natural Science Foundation of Guangxi (2016 GXNSFAA380194).

\section{Conflicts of Interest}

The authors declare no conflicts of interest regarding the publication of this paper. 


\section{References}

[1] Heggerud, C. and Lan, K. (2015) Local Stability Analysis of Ratio-Dependent Predator-Prey Models with Predator Harvesting Rates. Applied Mathematics and Computation, 270, 349-357. https://doi.org/10.1016/j.amc.2015.08.062

[2] Liu, M. and Wang, K. (2012) On a Stochastic Logistic Equation with Impulsive Perturbations. Computers \& Mathematics with Applications, 63, 871-886. https://doi.org/10.1016/j.camwa.2011.11.003

[3] Li, Y. and Gao, H. (2008) Existence, Uniqueness and Global Asymptotic Stability of Positive Solutions of a Predator-Prey System with Holling II Functional Response with Random Perturbation. Nonlinear Analysis, 68, 1694-1705. https://doi.org/10.1016/j.na.2007.01.008

[4] Ji, C., Jiang, D. and Li, X. (2010) Qualitative Analysis of a Stochastic Ratio-Dependent Predator-Prey System. Journal of Computational and Applied Mathematics, 235, 1326-1341. https://doi.org/10.1016/j.cam.2010.08.021

[5] Zuo, W. and Jiang, D. (2016) Stationary Distribution and Periodic Solution for Stochastic Predator-Prey Systems with Nonlinear Predator Harvesting. Communications in Nonlinear Science and Numerical Simulation, 36, 65-80. https://doi.org/10.1016/j.cnsns.2015.11.014

[6] Ji, C., Jiang, D. and Shi, N. (2009) Analysis of a Predator-Prey Model with Modified Leslie-Gower and Holling-Type II Schemes with Stochastic Perturbation. Journal of Mathematical Analysis and Applications, 359, 482-498. https://doi.org/10.1016/j.jmaa.2009.05.039

[7] Zhang, Y., Chen, S.H., Gao, S.J. and Wei, X. (2017) Stochastic Periodic Solution for a Perturbed Non-Autonomous Predator-Prey Model with Generalized Nonlinear Harvesting and Impulses. Physica A, 486, 347-366.

https://doi.org/10.1016/j.physa.2017.05.058

[8] Lu, C. and Ding, X. (2014) Persistence and Extinction of a Stochastic Logistic Model with Delays and Impulsive Perturbation. Acta Mathematica Scientia, 34B, 1551-1570. https://doi.org/10.1016/S0252-9602(14)60103-X

[9] Cheng, S. (2009) Stochastic Population Systems. Stochastic Analysis and Applications, 27, 854-874. https://doi.org/10.1080/07362990902844348

[10] Ji, C. and Jiang, D. (2009) Analysis of a Predator-Prey Model with Modified Leslie-Gower and Holling-Type II Schemes with Stochastic Perturbance. Journal of Mathematical Analysis and Applications, 359, 482-498.

https://doi.org/10.1016/j.jmaa.2009.05.039

[11] Ji, C. and Jiang, D. (2011) Dynamics of a Stochastic Density Dependent Predator-Prey System with Beddington-DeAngelis Functional Response. Journal of Mathematical Analysis and Applications, 381, 441-453. https://doi.org/10.1016/j.jmaa.2011.02.037

[12] Zhang, S.W. and Tan, D.J. (2015) Dynamics of a Stochastic Predator-Prey System in a Polluted Environment with Pulse Toxicant Input and Impulsive Perturbations. Applied Mathematical Modelling, 39, 6319-6331. https://doi.org/10.1016/j.apm.2014.12.020

[13] Zuo, W.J. and Jiang, D.Q. (2016) Periodic Solutions for a Stochastic Non-Autonomous Holling-Tanner Predator-Prey System with Impulses. Nonlinear Analysis, 22, 191-201. https://doi.org/10.1016/j.nahs.2016.03.004

[14] Li, D. and Xu, D. (2013) Periodic Solutions of Stochastic Delay Differential Equations and Applications to Logistic Equation and Neural Networks. Journal of the 
Korean Mathematical Society, 50, 1165-1181.

https://doi.org/10.4134/JKMS.2013.50.6.1165

[15] Liu, Z.J., Guo, S.L., Tan, R.H. and Liu, M. (2016) Modeling and Analysis of a Non-Autonomous Single-Species Model with Impulsive and Random Perturbations. Applied Mathematical Modelling, 40, 5510-5531.

https://doi.org/10.1016/j.apm.2016.01.008

[16] Higham, D.J. (2001) An Algorithmic Introduction to Numerical Simulation of Stochastic Differential Equations. SIAM Review, 43, 525-546.

https://doi.org/10.1137/S0036144500378302 